\title{
TALLANDO DESDE LO ALTO: UN SITIO DE EXPLOTACIÓN DE MATERIALES LÍTICOS DE LOS GRUPOS CAZADORES-RECOLECTORES DE LA ISLA GRANDE DE TIERRA DEL FUEGO
}

\author{
MYRIAN ALVAREZ", NÉLIDA PAL", LORENA SALVATELLI"***, IVAN BRIZ i GODINO+, \\ FRANCISCO A. ZANGRANDO ${ }^{++}$, ANDRÉS BAZERQUE ${ }^{++}$, HERNÁN DE ANGELIS ${ }^{* * *}$ Y DÁNAE FIORE ${ }^{\varnothing}$
}

\begin{abstract}
RESUMEN
En este trabajo se presentan los resultados iniciales obtenidos del análisis de un taller lítico, denominado Altos del Varela I, ubicado en la cima del cerro Petersen en la isla Grande de Tierra del Fuego (Argentina). El sitio presenta diferentes áreas que incluyen, de manera exclusiva, una alta frecuencia de lascas de riolitas metamorfizadas de origen local. Los resultados permiten abrir el debate sobre el uso de los paisajes montañosos por parte de las sociedades cazadoras-recolectoras fueguinas así como sobre la interacción costa-interior.
\end{abstract}

PALABRAS CLAVES: Taller lítico, Tierra del Fuego, paisaje montañoso, cazadores-recolectores.

\section{KNAPPING ON THE HILL: A LITHIC WORKSHOP SITE OF HUNTER- GATHERER GROUPS FROM TIERRA DEL FUEGO ISLAND}

\begin{abstract}
This paper provides the initial results obtained from the analysis of a lithic workshop, named Altos del Varela I, located on the top of Petersen hill in Tierra del Fuego Island (Argentina). The site contains distinctive areas entirely composed of a high frequency of flakes made from local metamorphic rhyolite. These results open the debate about the use of mountain landscape between the fuegian hunter-gatherers as well as the interaction between coastal and inland groups.

KEYWORDS: Lithic workshop, Tierra del Fuego, mountain landscape, hunter-gatherers.

\footnotetext{
* CONICET-CADIC. Bernardo Houssay 200. 9410 Ushuaia. Argentina. E-mail: myrianalvarez@gmail.com.

* CONICET-CADIC/INCUAPA. Bernardo Houssay 200. 02901. Ushuaia. Argentina. E-mail: nelidapal@yahoo.com.ar

*** ANPCyT-CADIC. Bernardo Houssay 200. 02901. Ushuaia. Argentina. E-mail: 1salvatelli@gmail.com

+ ICREA Researcher at IMF-CSIC. C/Egipcíaques 15. 08001. Barcelona. España. E-mail: ibriz@imf.csic.es. Ass. Researcher. Dept. of Archaeology. University of York. York. United Kingdom.

++ CONICET-CADIC/UBA. Bernardo Houssay 200. 02901. Ushuaia. Argentina. E-mail: panchozan@yahoo.com.ar

+++ Universidad de Buenos Aires, estudiante. E-mail: andresbazerque@hotmail.com

ø CONICET-AIA/UBA. Bartolomé Mitre 11317 “G”. 1036. Buenos Aires. Argentina. E-mail: danae fiore@yahoo.es
} 


\section{INTRODUCCIÓN}

Las estrategias de aprovisionamiento y explotación de materias primas líticas constituyen un tema central en las investigaciones sobre la organización tecnológica de las sociedades pasadas (Binford 1979; Démars 1982; Terradas 1996). La identificación de las fuentes de aprovisionamiento y la distribución de los productos que se generan en las distintas etapas de producción de los artefactos, han permitido determinar los ejes de circulación de las materias primas, sus territorios de explotación, las vías de desplazamiento de los grupos cazadoresrecolectores y las modalidades de ocupación de espacio (Geneste 1992). La localización de canteras y talleres de transformación de materiales líticos constituye el primer eslabón para comprender los procesos de gestión de recursos líticos y la generación del espacio social a nivel regional. El estudio de este tipo de yacimientos generalmente presenta, sin embargo, la dificultad de la ausencia de materiales fechables (Flegenheimer y Bayón 2002).

Dentro de las investigaciones realizadas en la isla Grande de Tierra del Fuego, las evidencias de talleres de producción lítica son especialmente escasas hasta la fecha (cfr. Borrero 1986; Piana et al. 1986). La información disponible sugiere que la mayor parte de las actividades productivas se concentraban en los asentamientos, donde fueron identificadas gran parte del procesado de las materias primas líticas (Terradas 1996; Mansur et al. 2000;
Borrazo 2004; Salemme et al. 2007; entre otros). Es por ello que en el marco del proyecto que investiga la variabilidad temporo-espacial de las ocupaciones de la región del Canal Beagle (PICT 2071) se propone ampliar la escala espacial de análisis e incluir sitios alejados de la costa.

En este trabajo, se dan a conocer los primeros resultados de la identificación y documentación del sitio Altos del Varela I. El sitio, constituido por una amplia área de producción de materiales líticos, está localizado sobre la cima del cerro Petersen (Figura 1). El emplazamiento del sitio en el interior montañoso abre la posibilidad de explorar la ocupación y generación del paisaje por parte de las sociedades fueguinas que ocuparon el interior de la isla y la costa del Canal Beagle; de esta forma se genera una interesante vía de investigación sobre los posibles límites de la apropiación del territorio, así como de los ámbitos de interrelación e interacción entre los diferentes grupos de la isla.

\section{APROVISIONAMIENTO Y EXPLOTACIÓN \\ DE MATERIAS PRIMAS EN LA ISLA GRANDE DE TIERRA DEL FUEGO: ANTECEDENTES GENERALES}

La acción de procesos erosivos de origen glaciar y glacifluvial ocurridos durante el Cuaternario tuvieron una fuerte incidencia en la conformación y la distribución de los recursos líticos en Tierra del Fuego. La mayor parte de rocas empleadas para la
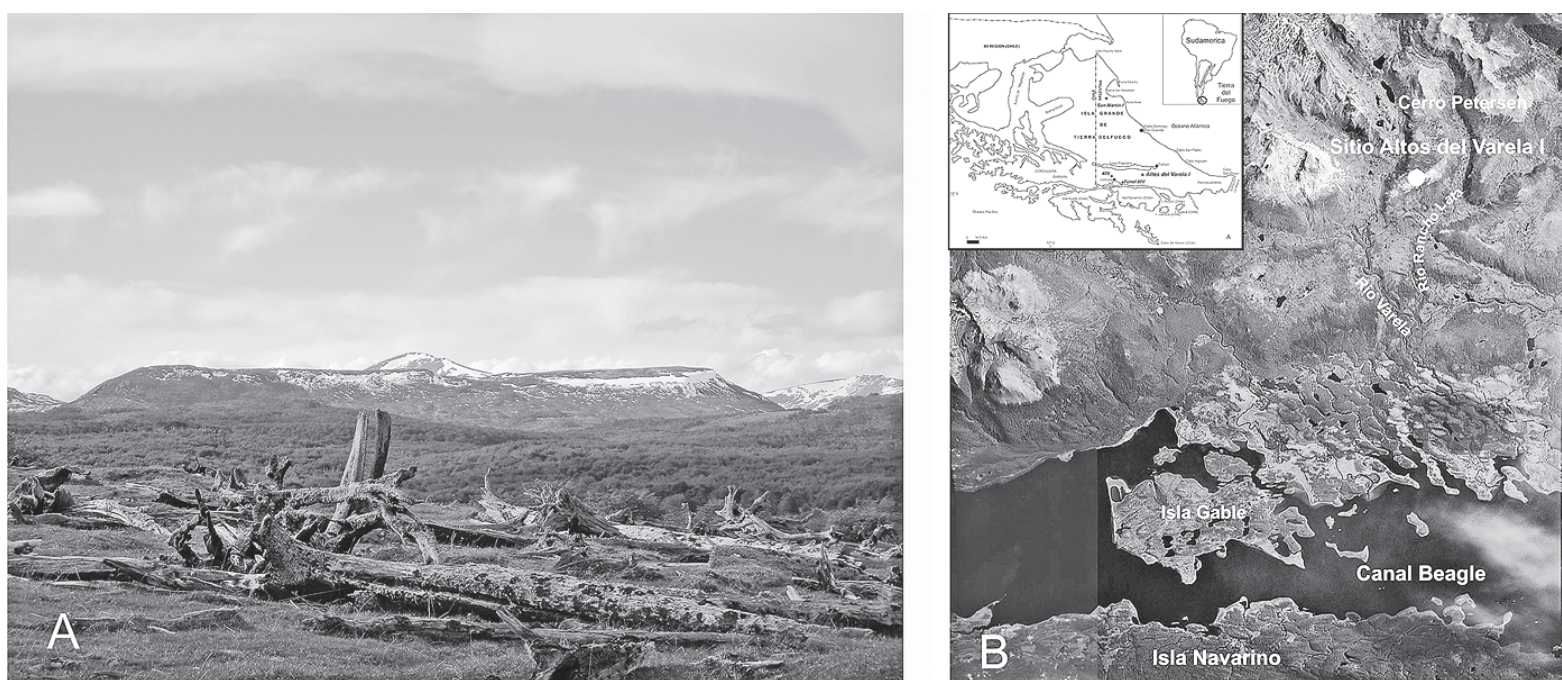

Fig. 1. A- Vista desde la costa del Cerro Petersen. B- Localización del sitio Altos del Varela I. 
talla proceden de depósitos secundarios o unidades sedimentarias más jóvenes, integradas por materiales redepositados sobre playas y cortes morrénicos próximos a las costas.

Las investigaciones realizadas sobre los grupos cazadores-recolectores-pescadores litorales que habitaron la costa norte del canal Beagle, indican que el 90\% de las rocas explotadas son de origen local, procedentes de la Formación Lemaire, tales como riolita, lutita, sílice (chert con radiolarios), tobas y arcilitas (Terradas 1996; Alvarez 2004).

Las evidencias disponibles para estas poblaciones indican que el aprovisionamiento de materias primas se habría realizado en fuentes secundarias localizadas a lo largo de la costa (Terradas 1996). Las actividades de procesamiento de materiales líticos era llevada cabo en los asentamientos y tan sólo en algunos casos, la etapa de descortezamiento inicial se habría realizado en los lugares de obtención. La única excepción a esta dinámica es el sitio Túnel XIV (Piana et al. 1986).

Entre los grupos cazadores-recolectores de ámbito terrestre que ocuparon en el centro y norte de la isla se observan tendencias similares en lo que respecta a las estrategias de obtención y explotación de materias primas líticas. El aprovisionamiento habría sido realizado en fuentes secundarias. Gran parte de estas rocas explotadas provienen de la Formación Lemaire y los procesos de producción eran realizados mayoritariamente en los asentamientos (Mansur et al. 2000; Borrazo 2004). Tan sólo el sitio San Martin 1 presenta indicios de actividades vinculadas con una cantera-taller (Borrero 1986:113).

\section{LOCALIZACIÓN Y EMPLAZAMIENTO DEL SITIO}

El sitio Altos del Varela I se ubica sobre el

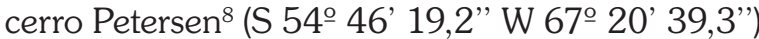
aproximadamente a los 600-700 msnm y a $10 \mathrm{~km}$ en línea recta de la costa actual, este cerro constituye un punto singular del paisaje, fácilmente identificable por su cumbre mesetiforme (Figura 1). Desde

8 El cerro fue denominado recientemente bajo ese nombre en honor al geólogo que realizó los primeros trabajos en la zona.
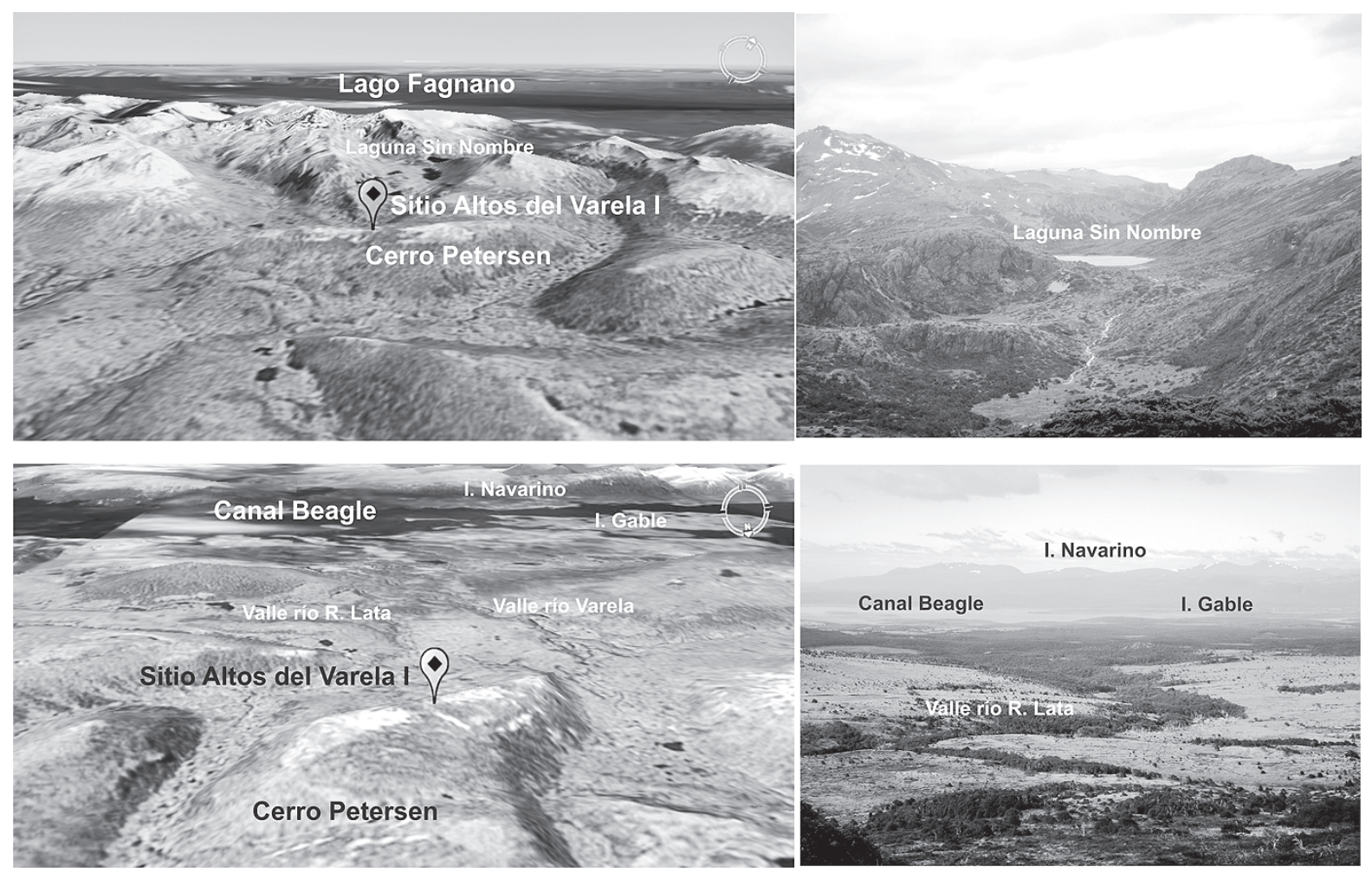

Fig. 2. Vista desde el sitio. Arriba de Sur a Norte hacia el interior de la isla. Abajo de Norte a Sur hacia el canal Beagle. 
la cima se observa el canal Beagle y los valles que conducen al interior montañoso estableciendo un amplio dominio visual del paisaje (Figura 2). El cerro Petersen es parte de la Formación Lemaire y si bien los afloramientos presentan una amplia distribución en la isla Grande de Tierra del Fuego, el valle del río Varela es, en la práctica, el único acceso directo a la Formación Lemaire desde este tramo de la costa, ya que en la mayor parte de su extensión se encuentra interrumpida por la Formación Yaghán, que se extiende de forma paralela al litoral.

El yacimiento se ubica en un sector deprimido e intermedio de la cumbre, en el límite de vegetación actual. El bosque circundante es achaparrado y presenta una distribución desigual; en el sustrato se observa un suelo de características minerales con la presencia de plantas rastreras y de trama muy cerrada. La mayor parte del sustrato se encuentra sin vegetación, con afloramientos de clastos y rocas.

\section{CARACTERÍSTICAS DEL SITIO, MÉTODOS DE TRABAJO Y RESULTADOS}

El sitio fue descubierto en 2006 durante un relevamiento geológico. Posteriormente, se llevaron a cabo dos intervenciones arqueológicas (2008) cuyos objetivos fueron: a) documentar el sitio; b) realizar diferentes sondeos de evaluación; c) muestrear los materiales arqueológicos, para esbozar los modelos de producción de artefactos líticos; d) detectar otras evidencias antrópicas en las inmediaciones y e) extraer una sección de suelo para posibles métodos de datación del contexto arqueológico.

El sitio está constituido por abundante material lítico tallado que se distribuye de forma discontinua sobre la superficie del cerro, por lo cual se pudieron delimitar cuatro sectores de diferente extensión (A, $\mathrm{B}, \mathrm{C}$ y D) (Figura 3). Se realizaron 4 sondeos de 20 x 20 x $11 \mathrm{~cm}$ en distintos puntos que posibilitaron obtener una interesante contextualización inicial: la generación del manto vegetal es posterior a la presencia del material arqueológico. El material lítico se distribuye desde la base del suelo, entre los 7 y $9 \mathrm{~cm}$ de profundidad. En el sector A, los artefactos emergen entre la vegetación en posición vertical, muy probablemente debido a los procesos de congelamiento-descongelamiento del suelo (Coronato com. pers.).

Pese a estar ubicado en la Formación Lemaire, se destaca la ausencia de bloques o afloramientos de riolitas en las proximidades del sitio. Los afloramientos del área corresponden a rocas de la Formación Lemaire pero facie sedimentaria, y a gabros de naturaleza intrusiva (González Guillot y Martinioni com pers.), no empleadas para los procesos de producción de artefactos líticos.

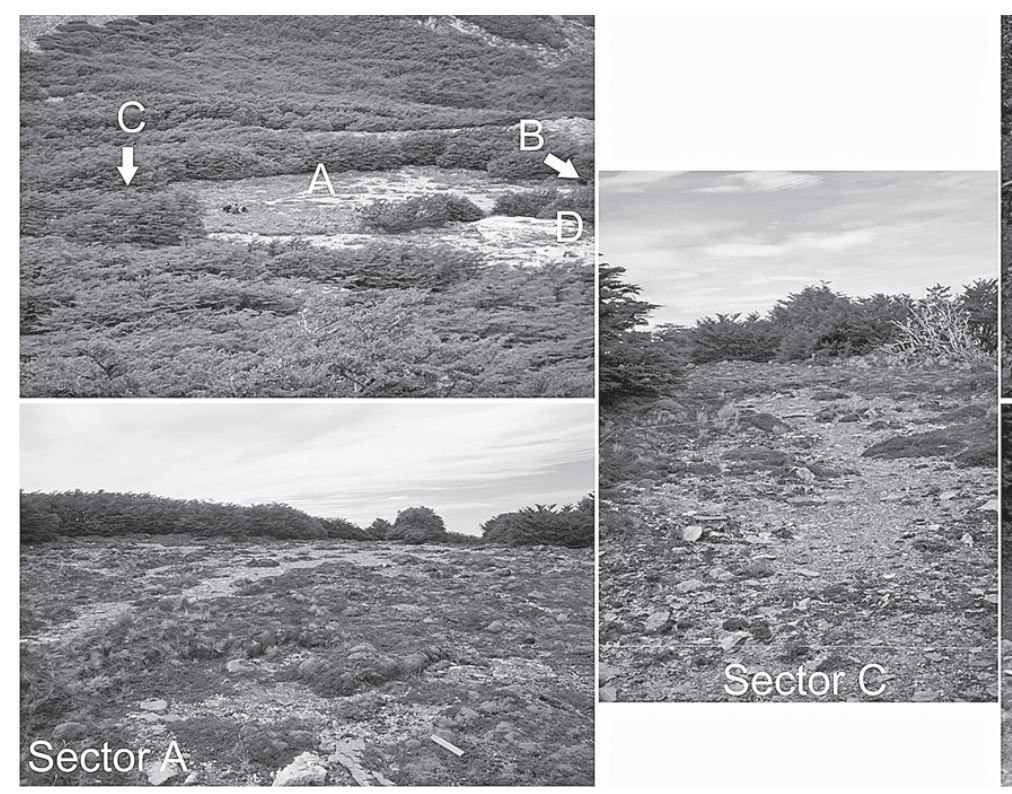

Fig. 3. Sectores del taller.

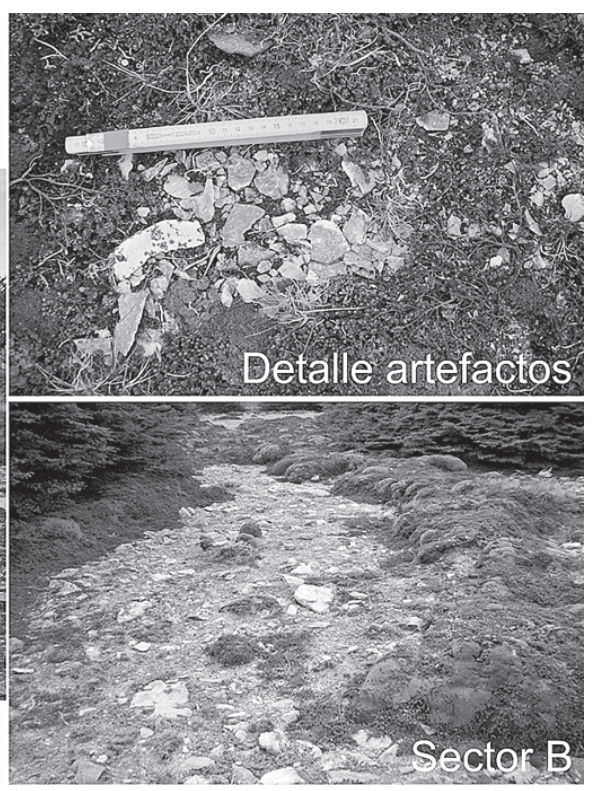


Debido a las dificultades de accesibilidad al sitio y a la gran cantidad de materiales presentes se priorizó la realización de la planimetría y muestreo del conjunto lítico. Cada una de las muestras implicó la recolección de los artefactos líticos presentes en una superficie de $1 \mathrm{~m}^{2}$. En el sector A se muestrearon 8 zonas aleatorias de $1 \mathrm{~m}^{2}$ y un sondeo de 20 x 20 $\mathrm{cm}$. En los sectores B y D se tomó una muestra de $1 \mathrm{~m}^{2}$ de cada uno de y en el sector $\mathrm{C}$ se recolectó la totalidad del material en superficie (Figura 3). La muestra total recuperada se compone de 3501 artefactos.

El conjunto incluye, fundamentalmente, lascas manufacturadas sobre riolitas $y$, en menor proporción, sobre tobas de grano fino metamorfizadas. La distribución por tamaños es variable, se hallaron desde microlascas hasta lascas de tamaños comprendidos entre los 8 y $12 \mathrm{~cm}$. La gran mayoría de ellas son resultado de talla por percusión directa; se observan abundantes lascas de reformatización de núcleos y hay indicios de trabajo bifacial.

Los artefactos no muestran el desarrollo de corteza característico de los materiales rodados por acción fluvio-glaciar, sino que presentan atributos que sugieren la extracción de formas base a partir de la explotación de bloques. Los tres núcleos registrados en superficie corroboran esta afirmación. No se registró la presencia de materiales formatizados por retoque. Algunas de las concentraciones exhiben una identidad litológica notable, lo que permite presuponer que corresponden a un mismo evento de talla.

Los artefactos no exhiben rodamiento o pátinas. Es llamativa la conservación de bordes y aristas en un área con alta exposición a la acción eólica. Esta buena preservación podría apuntar hacia una cronología reciente, pero por el momento no se poseen evidencias datables de forma independiente.

\section{REFLEXIONES FINALES}

Los resultados actuales indican que el sitio es un taller donde se llevó a cabo la producción de artefactos. Con respecto a su emplazamiento, se lo puede vincular con la caza del guanaco que es el único recurso faunístico presente en el cerro. Otras causas son difíciles de apuntar dado que la materia prima lítica allí disponible podría ser obtenida en cotas más bajas. Es llamativa la ausencia de afloramientos in situ que evidencien los primeros estadios del proceso productivo allí desarrollado, así como las áreas fuente de obtención de la materia prima mencionada.

Uno de nuestros primeros interrogantes fue tratar de identificar si los materiales generados fueron manufacturados por grupos marítimos o terrestres. En este sentido, el estudio de las estrategias de explotación y uso de las rocas (ambas sociedades usaban la misma materia prima lítica) y el análisis de la producción lítica no ofrecen una solución.

No obstante, las fuentes escritas brindan datos interesantes para aproximarnos al registro arqueológico. Lucas Bridges (2008:274 [1948]) señala que la gente Selk'nam conocía muy bien el paso cordillerano desde Harberton al lago Kami. Sin embargo, también el diario de Thomas Bridges indica partidas de caza del guanaco entre la gente Yámana, con campamentos de caza, a partir de los cuales recorrían grandes extensiones (Bridges 1878:12).

Más allá de esta discusión, la importancia de este trabajo radica en que Altos del Varela es uno de los pocos sitios-taller de la región, a la vez que evidencia la ocupación del interior montañoso y abre las puertas para discutir la interacción interior-litoral. Hasta el momento se cuenta con poca información que permita explicitar la dinámica de vínculos entre la costa y el interior; el único ejemplo es el sitio 409, localizado dentro del Parque Nacional de Tierra del Fuego (Monte Martial). La distancia respecto a la costa y su altura sobre el nivel del mar son elementos que permiten vincular ambos sitios (Piana y Martinioni 1998).

Para finalizar, la presencia de sitios arqueológicos en contextos montañosos nos conduce a replantear la visión del territorio por los grupos cazadores-recolectores pedestres y canoeros de Tierra del Fuego, quizás mucho más permeable y socialmente dinámicas, que nuestra mirada de barreras infranqueables.

\section{AGRADECIMIENTOS}

Queremos agradecer a Patricia Escola y Estela Mansur por las sugerencias vertidas para la realización de los trabajos de campo. A Karen Borrazo por sus comentarios y ayuda en la preparación de este trabajo. Juan Pérez contribuyó significativamente en la localización del sitio. Ernesto Piana nos proporcio- 
nó la información sobre el sitio 409. Agradecemos también a Andrea Coronato, Mauricio González Guillot y Daniel Martinioni por sus observaciones; Fernando Santiago prestó su colaboración para el desarrollo de este artículo. Natalie Goodall, Mónica Torres y la gente del Museo Akatushun nos brindaron su hospitalidad durante los trabajos de campo. Este proyecto fue financiado por un subsidio de la Agencia Nacional de Promoción Científica y Tecnológica (PICT 2071).

\section{BIBLIOGRAFÍA}

ALVAREZ, M. 2004. Estrategias tecnológicas en los grupos canoeros tempranos del área Fuego-Patagónica. Magallania 32:191-208.

BINFORD, L. 1979. Organization and formation processes: Looking at curated technologies. Journal of Archaeological Research 35(3):255-273.

BORRAZO, K. 2004. Tecnología lítica y uso del espacio en la costa norte fueguina. En: Temas de Arqueología. Arqueología del norte de la Isla Grande de Tierra del Fuego, editado por L. Borrero y R. Barberena, pp.5586. Editorial Dunken, Buenos Aires.

BORRERO, L. A. 1986. La economía prehistórica de los habitantes de la Isla Grande de Tierra del Fuego. Tesis doctoral inédita. Facultad de Filosofía y Letras. Universidad de Buenos Aires, Buenos Aires.

BRIDGES, L. 2008 [1948]. El último confín de la Tierra. Editorial Sudamericana, Buenos Aires.

BRIDGES, T. 1878. Cartas y fragmentos de su diario personal publicados en South American Missionary Magazine, Londres.

DEMARS P.Y. 1982. L'utilisation du silex au Paléolithique Supérieur: choix, approvisionement, circulation. L'exemple du bassin du Brive, Cahiers du Quaternaire n 5, CNRS, Bourdeaux.

FLEGENHEIMER, N Y C. BAYON. 2002. Cómo, Cuándo y Dónde? Estrategias de abastecimiento lítico en la pam- pa bonaerense. En: Del Mar a los Salitrales. Diez mil Años de Historia Pampeana en el Umbral del Tercer Milenio, editado por D. Mazzanti, M. Berón y F. Oliva), pp.: 301-309. Facultad de Humanidades, Universidad de Mar del Plata, Mar del Plata.

GENESTE, J. 1992. L'aprovisionnement en matières premières dans les systemes de production: la dimension spatiale de la tecnologie. Tecnología y Cadenas Operativas Líticas. Treballs d' Arqueologia I: 2-35.

MANSUR, M.; D. MARTINIONI y A. LASA. 2000. La gestión de recursos líticos en el sitio Marina I. En: Desde el País de los Gigantes. Perspectivas Arqueológicas en Patagonia, editado por J. B. Belardi, F. Carballo Marina y S. Espinosa, pp,: 57-72. Universidad Nacional de la Patagonia Austral, Río Gallegos.

PIANA, E.; G. CANALE y A. CORONATO. 1986. Túnel XIV: el núcleo de la cuestión. Morenas y materia prima lítica en el Canal Beagle. Trabajo presentado en el XI Congreso Nacional de Arqueología Argentina, San Rafael, Mendoza.

PIANA, E. Y D. MARTINIONI. 1998. Informe sobre un yacimiento arqueológico en el valle de Andorra. Jurisdicción del Parque Nacional Tierra del Fuego. Centro Austral de Investigaciones Científicas (CONICET-CADIC), Ushuaia. Ms.

SALEMME, M., G. BUJALESKY. y F. SANTIAGO. 2007. La Arcillosa 2: la ocupación humana durante el Holoceno medio en el Río Chico, Tierra del Fuego, Argentina. En: Arqueología de Fuego-Patagonia. Levantando piedras, desenterrando huesos... y develando arcanos. Editado por F. Morello, M. Martinic, A. Prieto y G. Bahamonde, pp., 723-736. Ediciones CEQUA, Punta Arenas (Chile).

TERRADAS, X. 1996. La gestió dels recursos minerals entre les comunitatas caçadores-recol-lectores. Vers una representació de les estratègies de proveïment de matèries primeres. Tesis de doctorado inédita. Universidad Autónoma de Barcelona, Barcelona. 